Urologe $2019 \cdot 58: 802-803$

https://doi.org/10.1007/s00120-019-0948-8

Online publiziert: 10. Mai 2019

(c) Springer Medizin Verlag GmbH, ein Teil von Springer Nature 2019

\section{J. Steffens}

Klinik für Urologie und Kinderurologie, St. Antonius-Hospital gGmbH, Eschweiler, Deutschland
Seit 1996 bin ich Chefarzt der Klinik für Urologie und Kinderurologie des St. Antonius-Hospitals gGmbH in Eschweiler, einem akademischen Leerkrankenhaus der RWTH Aachen. Damit erfüllte ich mir einen beruflichen Lebenstraum und wurde Nachfolger meines Vaters in gleicher Position. Dieser gründete die Abteilung 1961 als Belegabteilung und 1967 als Hauptabteilung. Meine breite urologische Ausbildung erhielt ich an den Universitätskliniken Berlin und Homburg/Saar sowie bei meinem Vater. Neben meiner Kliniktätigkeit übte ich 10 Jahre lang ehrenamtliche Tätigkeiten im Berufsverband der Deutschen Urologen (BDU) und in der Deutschen Gesellschaft für Urologie (DGU) aus. Sechs Jahre war ich Vorsitzender des Arbeitskreises der leitenden Krankenhausärzte im BDU. Höhepunkt meiner berufspolitischen Karriere war meine DGU-Präsidentschaft 2011. Als stellvertretender Vorsitzender leite ich die Juniorakademie der Deutschen Urologen und bin aktiv in der Arbeitsgemeinschaft "Junge Urologen“ der DGU tätig. Mein berufspolitisches Hauptanliegen betrifft die Nachwuchsförderung junger Urologen.

Medizin ist nicht nur eine Natur-, sondern auch eine Menschenwissenschaft. Deshalb bereitet mir nicht nur das operative Handwerk mit Einsatz moderner Techniken, sondern auch der Umgang mit den Menschen große Freude. Dies betrifft einerseits das Verständnis des Kranken in seinen Ausnahmesituationen, die Bewältigung von durch Krank- heit verursachten Lebenskrisen und die schrittweise Betreuung bei der Krankheitsbewältigung. Andererseits erfüllt mich die Führung meiner Mitarbeiter mit Stolz und Dankbarkeit. Die Persönlichkeitsentwicklung begabter junger Urologinnen/Urologen, die Förderung unterschiedlicher Talente und Hilfen bei der Karriereplanung machen meinen Arbeitsalltag abwechslungsreich.

Problematisch ist in einer ökonomisch strukturierten Welt der Rückzug als persönlich für den Patienten verantwortlicher Arzt. Merkantile Anreize für eine erfolgreiche Tätigkeit, die Einhaltung vereinbarter Jahresziele und der ökonomische Druck in den Krankenhäusern haben $\mathrm{zu}$ einer Verlagerung chefärztlicher Interessen geführt. Während die qualitativ angemessene Versorgung der Patienten selbstverständlich hingenommen wird, besteht von Verwaltungsseite die gestiegene Erwartungshaltung durch Steigerung der Patientenzahlen und des Case-mix-Indexes. Aufgrund solider Patientenversorgung, selbstkritischem Umgang mit komplikationsträchtigen Behandlungsverläufen und guter intersektoraler Kommunikation mit den Niedergelassenen bin ich stolz auf eine funktionstüchtige stabile Abteilung. Vor dem Hintergrund guter Zahlen versuche ich durch Vorbildfunktion in meinem Team den Eindruck zu vermitteln, dass wir nicht seelenlose Akteure in einer rein merkantilen Gesundheitsbranche sind, sondern den Menschen in den Mittelpunkt stellen und ihn so behandeln, als ob wir selbst betroffen wären.

\section{Bürgerversicherung zu erwarten}

Als angestellter Chefarzt mit altem Vertrag stört mich das Vertragssystem. Das Schielen nach Privatpatienten ist notwendig, aber lästig. Mein Einkommen wird jedoch ausschließlich durch die Einnahmen aus der privatärztlichen Liquidation erzielt, so dass ich nur mit diesen Eingriffen Einnahmen erwirtschafte. Wünschenswert wäre ein ausreichendes Festhonorar ohne ökonomische Antriebe und Zielvereinbarung. Voraussetzung wäre eine ausreichend gute finanzielle Ausstattung, die jedoch bei neuem Vertragsabschluss nicht gewährt würde. Gegenwärtig ist eine Herabstufung der Chefarztgehälter zu erkennen, was die Eigenverantwortung leitender Krankenhausärzte nicht steigert. Bei einer zu erwartenden Bürgerversicherung in der nächsten Legislaturperiode werden auch finanzielle Verschiebungen verantwortlich tätiger leitender Krankenhausärzte zu erwarten sein. Vor diesem Hintergrund könnte es schwierig werden, die Motivation qualifizierter Oberärzte mit chefärztlichen Ambitionen zu steigern. Gespräche mit der Geschäftsführung des Krankenhauses mit dem Ziel einer guten Dotierung für einen Nachfolger sind wichtig, um eine funktionierende Abteilung zukunftsfähig zu machen.

Die Suche nach einem qualifizierten, das Fach beherrschenden Chefarzt und die Zusammenstellung eines primär menschenorientiert handelnden und nicht nur ökonomischen Zwängen 
verhafteten Teams sehe ich als größte Herausforderung für die künftige ärztliche Tätigkeit im Krankenhaus. Urologische Patienten werden aufgrund des demographischen Wandels auch künftig in ausreichender Zahl zu versorgen sein.

\section{Sprechende Medizin unwirtschaftlich}

Unter dem ökonomischen Druck werden heute wirtschaftlich lukrative Leistungen häufiger durchgeführt als schlecht vergütete Maßnahmen der „sprechenden Medizin“. Der zeitliche Aufwand für die Patientengespräche wird im System der Fallpauschalen nicht berücksichtigt. Künftig sollten Arzt/Patienten-Gespräche und interdisziplinäre Fachgespräche in die Berechnung einfließen. Dies ist auch deshalb wichtig, da Patienten zunehmend mehrfach und chronisch erkranken.

\section{Neues Rollenverständnis in der Krankenhausführung}

Die Rolle von Ärzten und Pflegenden wird und muss sich ändern. Deshalb halte ich eine gemeinsame Krankenhausführung, in der sowohl Ärzte als auch Pflegende mit kaufmännischen Direktoren auf Augenhöhe Entscheidungen treffen, für sinnvoll. Nur so ist ein Wertemanagement im Krankenhaus möglich, welches sich nicht nur an betriebswirtschaftlichen Zielen ausrichtet, sondern medizinische Maßnahmen und Patientenorientierung integriert. Eine bessere Verzahnung zwischen den Akteuren könnte auch zur Verbesserung der Arbeitsbedingungen führen.

\section{Qualitätseinbußen durch betriebswirtschaftlichen Erfolgsdruck}

Ursachen für die schwierigen Arbeitssituationen in den Krankenhäusern ist auch der ökonomische Erfolgsdruck mit einer Mengenausweitung aufgrund falscher Erlösanreize. Die Zahl der Krankenhausbetten ist trotz kürzerer Liegezeiten kaum gesunken, die Zahl der Behandlungsfälle jedoch deutlich gestiegen. Eine hohe Bettendichte in einem
Fach ist ein Treiber für die Durchschleusung hoher Fallzahlen. Dies geht zu Lasten der Qualität. Jedes Krankenhaus kann jeden Eingriff ohne Qualitätsnachweis durchführen. Eine Spezialisierung ist gegenwärtig noch ohne notwendige Erfahrung möglich. Die AWMF-Forderung zur qualifizierten Zentrumsbildung könnte bei manchen Krankheitsbildern die Versorgungsqualität steigern.

\section{Krankenhausvergütung nach Qualitätsindikatoren ausrichten}

Die Krankenhausplanung wird sich künftig am regionalen Bedarf orientieren und sektorenübergreifend ausrichten. Über-, Unter- und Fehlversorgung kann künftig nur gemeinsam von Politik, Krankenhausmanagement, Selbstverwaltung und Ärzteschaft sowie Patientenvertretern beseitigt werden.

$\mathrm{Zu}$ den Maßnahmen, die die Politik ergreifen muss, gehört eine Umgestaltung des DRG-Systems. Heute herrscht in den Krankenhäusern die Mentalität, die Therapie nach Quantität auszurichten, um dadurch eine höhere Vergütung zu erzielen. Ziel muss es sein, die Vergütung ebenfalls nach Qualitätsindikatoren auszurichten - auch, wenn solche Indikatoren in der Urologie noch erarbeitet werden müssen.

\section{Zusammenfassung}

Ziel unserer Bemühungen muss es sein, den Patienten nicht zum Kunden mutieren $\mathrm{zu}$ lassen und einen partnerschaftlichen Dialog zwischen Krankenhausverwaltung und Ärzteschaft anzustreben. Die Bedürfnisse des Patienten sollten stark in den Mittelpunkt rücken. Eine gute Arzt-Patienten-Beziehung wird auch künftig für eine erfolgreiche Versorgung unverzichtbar sein. Darüber hinaus wird ein Strukturwandel notwendig werden. Bei einer Bürgerversicherung muss eine ausreichend gute Dotierung des Chefarztes künftig der die Verantwortung tragenden Persönlichkeit gerecht werden. Stärkung der ärztlichen Kompetenz im Rahmen der Krankenhausführung, eine mehr am Bedarf orientierte Krankenhausplanung, Abbau von Überkapazitäten und Anpassung des DRG-basierten-Vergütungssystems werden Aufgaben der nahen Zukunft sein.

Fazit. Die Tätigkeit als Chefarzt ist bei hohem persönlichem Einsatz im Verbund mit guten oberärztlichen Vertretern und einem qualifizierten Team eine erfüllende Tätigkeit. Die Kommunikation mit Patient und Kollegen muss trotz steigendem Bürokratieaufwand persönlich eingefordert werden. Die Erzielung wirtschaftlich guter Geschäftszahlen gelingt leider nur im Verdrängungswettbewerb. Akzeptanz bei der geschäftsführenden Krankenhausleitung kann nur bei Erreichen guter Geschäftszahlen erwartet werden.

\section{Korrespondenzadresse

Prof. Dr. med. J. Steffens
Klinik für Urologie und
Kinderurologie, St. Antonius-
Hospital gGmbH
Dechant-Deckers-Str. 8,
52249 Eschweiler,
Deutschland
joachim.steffens@
sah-eschweiler.de

Prof. Dr. med. J. Steffens Chefarzt der Klinik für Urologie und Kinderurologie, Eschweiler. Prof. Joachim Steffens ist Arzt für Urologie mit den Zusatzbezeichnungen: Röntgendiagnostik Harntrakt, Medikamentöse Tumortherapie und Andrologie.

\section{Einhaltung ethischer Richtlinien}

Interessenkonflikt. J. Steffens gibt an, dass kein Interessenkonflikt besteht.

Für diesen Beitrag wurden von den Autoren keine Studien an Menschen oder Tieren durchgeführt. Für die aufgeführten Studien gelten die jeweils dort angegebenen ethischen Richtlinien. 